\title{
Adesão à terapia antirretroviral em pacientes HIV soropositivos no Brasil: uma revisão da literatura
}

\author{
Adherence to antiretroviral therapy in HIV-seropositive patients in Brazil: \\ a literature review

\section{Adhesión a la terapia antirretroviral en pacientes VIH seropositivos en Brasil: una revisión de literatura}

\author{
Cléa Adas Saliba GARBIN ${ }^{1}$ \\ Renata Colturato Joaquim GATTO ${ }^{2}$ \\ Artênio José Ísper GARBIN ${ }^{3}$ \\ ${ }^{1}$ Professora Titular do Programa de Pós-graduação em Odontologia Preventiva e Social. Faculdade de Odontologia, \\ UNESP Univ. Estadual Paulista, 16015-050 Araçatuba - SP, Brasil \\ ${ }^{2}$ Aluna de Doutorado do Programa de Pós-Graduação em Odontologia Preventiva e Social. Faculdade de Odontologia, \\ UNESP Univ. Estadual Paulista, 16015-050 Araçatuba - SP, Brasil \\ ${ }_{3}^{3}$ Professor Adjunto do Programa de Pós-graduação em Odontologia Preventiva e Social. Faculdade de Odontologia, \\ UNESP Univ. Estadual Paulista, 16015-050 Araçatuba - SP, Brasil
}

\begin{abstract}
Resumo
Introdução: As pessoas infectadas pelo vírus HIV têm acesso gratuito à terapia antirretroviral (TARV) no Brasil, no entanto, para que funcione é preciso que o paciente conduza a manutenção prolongada do tratamento, pois a efetividade da TARV depende diretamente da adesão do paciente. Objetivos: realizar uma revisão integrativa de literatura tendo como pergunta norteadora "Qual a taxa de não adesão à terapia antirretroviral de pacientes HIV soropositivos no Brasil?". Metodologia: Foram consultadas as seguintes bases de dados: Bireme, Pubmed, Medline, Scielo, Scopus, Web Of Science e Cochrane Library, entre os meses de maio e junho de 2016. Após a aplicação dos critérios de inclusão e exclusão, os seguintes dados foram extraídos dos artigos que compuseram o estudo: ano de publicação, região em que foram realizados, população de estudo, método de verificação da adesão e taxa de não adesão à TARV. Resultados: 11 artigos foram selecionados para compor a presente revisão, que datam de 2009 a 2015. Os estudos têm uma tendência a polarização em algumas regiões do país e a população participante, em sua maioria era adulta. O método para verificação da adesão mais utilizado foi a retirada do medicamento nas farmácias, seguido pela aplicação de questionário validado. A taxa de não adesão a TARV variou de $18 \%$ à 74,3\% nos artigos analisados. Conclusão: Houve um alto índice de não adesão à terapia antirretroviral nos estudos que participaram dessa revisão.
\end{abstract}

Descritores: Adesão à medicação; Antirretrovirais; HIV; Síndrome de Imunodeficiência Adquirida.

\begin{abstract}
Introduction: People infected with HIV have free access to antiretroviral therapy (ART) in Brazil, however, in order to work, it is necessary that the patient leads to prolonged maintenance treatment, since the effectiveness of antiretroviral therapy depends directly on patient compliance. Objectives: To carry out an integrative literature review using the following guiding question "What is the rate of non-adherence to antiretroviral therapy of HIV positive patients in Brazil?". Methodology: the following databases were consulted: Bireme, Pubmed, Medline, Scielo, Scopus, web of Science and the Cochrane Library, between the months of May and June 2016. After the application of inclusion and exclusion criteria, the following data were extracted from articles that composed the study: year of publication, the region in which they were conducted, population targeted, the membership verification method of adherence and rate of nonadherence to ART. Results: 11 articles were selected for the present review, dating from 2009 to 2015 . The studies have a tendency to focus in some regions of the country and the population targeted was mostly adult. The most used method for checking adherence was the withdrawal of the drug from pharmacies, followed by validated questionnaire. The rate of non-adherence to ART ranged from $18 \%$ to $74.3 \%$ in the studied articles. Conclusion: There was a high rate of nonadherence to antiretroviral therapy in the studies who participated in this review.
\end{abstract}

Descriptors: Medication Adherence; Anti-Retroviral Agents; HIV; Acquired Immunodeficiency Syndrome.

\begin{abstract}
Resumen
Introducción:Las personas infectadas por el virus VIH tienen acceso gratuito a la terapia antirretroviral (TARV) en Brasil, sin embargo, para que tenga efecto es necesario que el paciente mantenga la manutención prolongada del tratamiento, pues la efectividad de la TARV depende directamente de la adhesión del paciente. Objetivos: Realizar una revisión integral de literatura cuya pregunta norteadora es "cuál es la tasa de no adhesión a la terapia antirretroviral de pacientes VIH seropositivos en Brasil?". Metodología: Se consultaron las bases de datos Bireme, Pubmed, Medline, Scielo, Scopus, Web of Sciencey Cochrane Library entre los meses de mayo y junio de 2016. Tras la aplicación de criterios de inclusión y exclusión se extrajeron los siguientes datos de los artículos que compusieron este estudio: año de publicación, región en que se realizaron, población de estudio, método de verificación de la adhesión y tasa de no adhesión a la TARV. Resultados:Se seleccionaron 11 artículos para componer esta revisión fechados entre 2009 y 2015 . Los estudios tienen tendencia a la polarización en algunas regiones del país y la población participante es de adultos en su mayoría. El método más utilizado para verificación de la adhesión fue la periodicidad de retirada del medicamento en las farmacias y la aplicación de cuestionario validado. La tasa de no adhesión a la TARV varió entre el $18 \%$ y el 74,3\% en los artículos analizados. Conclusión: Se identificó un alto índice de no adhesión a la terapia antirretroviral en los estudios que compusieron esta revisión.

Descriptores: Adhesión a la medicación; Antirretrovirales; SIDA; VIH; Síndrome de Inmunodeficiencia Adquirida.
\end{abstract}

\section{INTRODUÇÃO}

Desde o início da epidemia de AIDS no Brasil, até junho de 2015, foram registrados no país 798.366 casos da doença. Embora a taxa de detecção de pessoas infectadas pelo vírus HIV tenha estabilizado na ultima década, cerca de 
0,6 mil novos casos são registrados por ano no país ${ }^{1}$.

O governo brasileiro dispõe de uma série de políticas públicas destinadas a população infectada pelo vírus. Logo nos primeiros anos da descoberta da doença, a legislação já garantia benefícios aos portadores da Síndrome da Imunodeficiência Adquirida - SIDA/AIDS, por meio do decreto de Lei $n^{\circ} 7.670 / 88$, que dispõe sobre a licença para tratamento médico, auxilio doença ou aposentadoria, pensão especial entre outros ${ }^{2}$. Atualmente, a legislação vigente estende-se a diversos setores do cotidiano, no que diz respeito à justiça, às finanças, ao trabalho, ao transporte e ainda, contra a discriminação. Recentemente, a LEI $\mathrm{N}^{\circ}$ 12.984, de 2 de junho de 2014, definiu como crime a discriminação dos portadores do vírus da imunodeficiência humana (HIV) e doentes de AIDS sob penalidade de reclusão e/ou multa ${ }^{3}$.

Campanhas de prevenção e conscientização da população são constantemente divulgadas pelo Ministério da Saúde. A população também tem acesso à informação em diversos sites governamentais e não governamentais, que disponibilizam informações importantes aos pacientes soropositivos, como locais de tratamento, medicamentos, efeitos colaterais, sintomas da doença, prevenção, transmissão, qualidade de vida, direitos, publicações científicas, cartilhas, entre outras.

Embora a taxa de mortalidade nacional tenha tido uma queda de 5,0\% nos últimos 10 anos, essa realidade não representa todas as regiões do país. $\mathrm{O}$ boletim epidemiológico sobre HIV/Aids no Brasil apresenta esses valores por região:

\begin{abstract}
“...essa redução não é observada em todas as regiões do país; apenas as regiões Sudeste e Sul apresentam tendências de queda, sendo que a região Sul teve redução de $10,6 \%$ e no Sudeste essa redução foi mais acentuada, de 19,7\%. Nas regiões Norte e Nordeste, a tendência é de crescimento nos últimos dez anos; no Norte, o coeficiente aumentou $58,6 \%$, passando-se de 4,6 óbitos para cada 100 mil habitantes em 2005 para 7,3 em 2014, e no Nordeste, aumentou $34,3 \%$, passandose de 3,2 para 4,3 óbitos para cada 100 mil habitantes. A região Centro-Oeste manteve o coeficiente de 4,5 em $2005 e$ em 2014."
\end{abstract}

Boletim epidemiológico Aids e DST ${ }^{1}$

Além disso, observa-se que do total dessas pessoas vivendo com HIV, $80 \%$ foram diagnosticadas e $48 \%$ estão em tratamento para o $\mathrm{HIV}^{1}$.

A política nacional garante ao paciente infectado, acesso gratuito ao tratamento em centros especializados. Em 2016, o país completa 20 anos de livre acesso à terapia antirretroviral altamente ativa (HAART), distribuída gratuitamente pelo Sistema Único de Saúde (SUS) para todas as pessoas que vivem com HIV / AIDS. O tratamento da AIDS no Brasil tem sido amplamente citado como o maior e mais bem sucedido programa de combate à AIDS entre os países em desenvolvimento ${ }^{4}$. $\mathrm{O}$ avanço tecnológico permitiu uma alteração do perfil da doença, que culturalmente era associada a morte anunciada e passou a apresentar um caráter crônico, possibilitando ao portador do vírus viver melhor com a doença e por mais tempo ${ }^{5}$.

No entanto, mesmo com a grande disponibilidade desses recursos terapêuticos, a literatura destaca o fato de alguns pacientes não se beneficiarem com essa condição. Isso porque para garantir o sucesso do tratamento com a terapia antirretroviral, um aspecto é essencial: a adesão ao tratamento ${ }^{6}$. Falhas ocasionais na adesão da TARV podem limitar os benefícios do tratamento ${ }^{7}$. Algumas dificuldades encontradas quanto à adesão decorrem, em parte, da complexidade do uso dessa terapia e dos efeitos colaterais advindos dela. Somando-se aos fatores de natureza biomédica que influenciam os níveis de adesão e resultados da TARV, aspectos psicossociais também podem interferir negativamente na colaboração do paciente ao uso da Terapia Antirretroviral $^{8}$.

Diante do exposto, objetivou-se verificar a taxa de não adesão à terapia antirretroviral, de pacientes portadores do vírus HIV, no cenário nacional.

\section{MATERIAL E MÉTODO}

Trata-se de uma revisão integrativa da literatura, na qual a pergunta norteadora foi: "Qual a taxa de não adesão à terapia antirretroviral de paciente HIV soropositivo no Brasil?". O estudo foi conduzido em 6 etapas: 1. Elaboração da pergunta norteadora; 2. Busca na literatura; 3. Coleta de dados; 4. Análise crítica dos estudos; 5. Discussão dos resultados; 6. Apresentação da revisão integrativa 9 .

O levantamento dos artigos científicos foi realizada nos meses de maio e junho de 2016, nas bases de dados: Bireme, Pubmed, Medline, Scielo, Scopus, Web Of Science e Cochrane Library, utilizando os descritores disponíveis no DECS: "Adesão à medicação", "Antirretrovirais", "HIV" e "Síndrome de Imunodeficiência Adquirida" e os correspondentes em inglês disponíveis no MESH: "Adherence Patient", "Anti-Retroviral Agents" e "Acquired Immunodeficiency Syndrome". Os operadores boleanos utilizados foram AND e OR.

Os critérios de inclusão foram: artigos completos disponíveis eletronicamente, nos idiomas português, inglês ou espanhol, que apresentassem ideia clara, objetiva e condizente com o tema abordado, publicados a partir de 1996, ano em que a terapia antirretroviral passou a ser distribuída gratuitamente pelo SUS. Os critérios de exclusão foram os artigos que não se adequavam ao tema e aqueles que os sujeitos da pesquisa não eram brasileiros. O quadro 1 apresenta os artigos que foram selecionados para compor o estudo.

\section{RESULTADOS}

Foram selecionados para compor a presente revisão 11 artigos científicos que datam de 2009 à 2015. O Gráfico 1 ilustra as regiões, nas quais, os estudos foram realizados, nota-se a ausência de pesquisas realizadas na região Norte e no Centro-Oeste.

O Quadro 2 apresenta uma síntese dos estudos que compõe a revisão. É possível observar os estudos divididos por região, pela população estudada, pelo método utilizado para verificar a adesão à TARV, e por fim, pela taxa de não adesão à Terapia Antirretroviral. Nota-se um alto índice de não adesão a TARV, independente da metodologia utilizada para a verificação da mesma.

\section{DISCUSSÃO}

Poucos estudos foram realizados sobre a adesão à terapia antirretroviral na população brasileira. O primeiro artigo foi realizado em 2009, mas a terapia é oferecida a população gratuitamente desde 1996.

Outro achado que chama a atenção é a distribuição dos estudos por região. Nenhuma pesquisa foi realizada no 
Centro-Oeste e no Norte do país, sendo esta última a região que teve a maior taxa de crescimento de mortalidade em decorrência da AIDS nos últimos 10 anos, onde o coeficiente aumentou em 58,6\% ${ }^{1}$. O Brasil apresenta um padrão heterogêneo de distribuição geográfica dos casos de
AIDS, decorrente da grande amplitude do território e da polarização de casos em determinadas regiões ${ }^{10}$. Na década de 2000, houve expansão dos casos nas regiões Norte e Nordeste, enquanto as demais macrorregiões do país apontaram estabilidade ${ }^{11}$

Quadro 1: Lista de artigos selecionados para compor a revisão.

\begin{tabular}{|l|l|l|l|l|}
\hline $\mathbf{N}$ & Autores & $\begin{array}{l}\text { Ano de } \\
\text { publicação }\end{array}$ & Cidade / Estado & Tipo de estudo \\
\hline 1 & $\begin{array}{l}\text { Gomes RR de FM, } \\
\text { Machado CJ, Acurcio F de A, Guimarães MDC. }\end{array}$ & 2009 & $\begin{array}{l}\text { Belo Horizonte/ } \\
\text { MG }\end{array}$ & Coorte \\
\hline 2 & Trombini ES, Schermann LB. & 2010 & $\begin{array}{l}\text { Canoas, } \\
\text { Cachoeira do Sul } \\
\text { e Passo Fundo / } \\
\text { RS }\end{array}$ & Descritivo Transversal \\
\hline 3 & $\begin{array}{l}\text { Rocha GM, Machado CJ, } \\
\text { Acurcio F de A, } \\
\text { Guimarães MDC. }\end{array}$ & $\begin{array}{l}\text { Belo Horizonte / } \\
\text { MG }\end{array}$ & Descritivo Transversal \\
\hline 4 & $\begin{array}{l}\text { Leite LHM, Papa A, Valentini RC. } \\
5\end{array}$ & $\begin{array}{l}\text { Felix G, Ceolim MF. } \\
\text { Rio de Janeiro / } \\
\text { RJ }\end{array}$ & $\begin{array}{l}\text { Descritivo } \\
\text { Transversal } \\
\text { interior / SP }\end{array}$ & Descritivo Transversal \\
\hline 6 & Camargo LA, Capitão CG, Filipe EMV. & 2011 & São Paulo / SP & Descritivo Transversal \\
\hline 7 & $\begin{array}{l}\text { Jacques IJAA, Santana JM, Moraes DCA, Souza } \\
\text { AFM } \\
\text { Abrão FMS, Oliveira RC. }\end{array}$ & 2014 & Pernambuco / PE & Descritivo Transversal \\
\hline 8 & $\begin{array}{l}\text { Silva ACO, } \\
\text { Reis RK, Nogueira JA, Gir E. }\end{array}$ & 2012 & João Pessoa / PA & Descritivo transversal \\
\hline 9 & $\begin{array}{l}\text { Moraes DCA, Oliveira RC, } \\
\text { Costa SFG. }\end{array}$ & 2014 & Pernambuco / PE & Descritivo Transversal \\
\hline 10 & $\begin{array}{l}\text { Tufano CS, Amaral RA, Cardoso RLC, Malbergier } \\
\text { A. }\end{array}$ & 2015 & São Paulo /SP & $\begin{array}{l}\text { Estudo transversal de } \\
\text { prevalência }\end{array}$ \\
\hline 11 & Silva JAG, Dourado I, Brito AM, Silva CAL. & 2015 & Salvador /BA & Descritivo Transversal \\
\hline
\end{tabular}

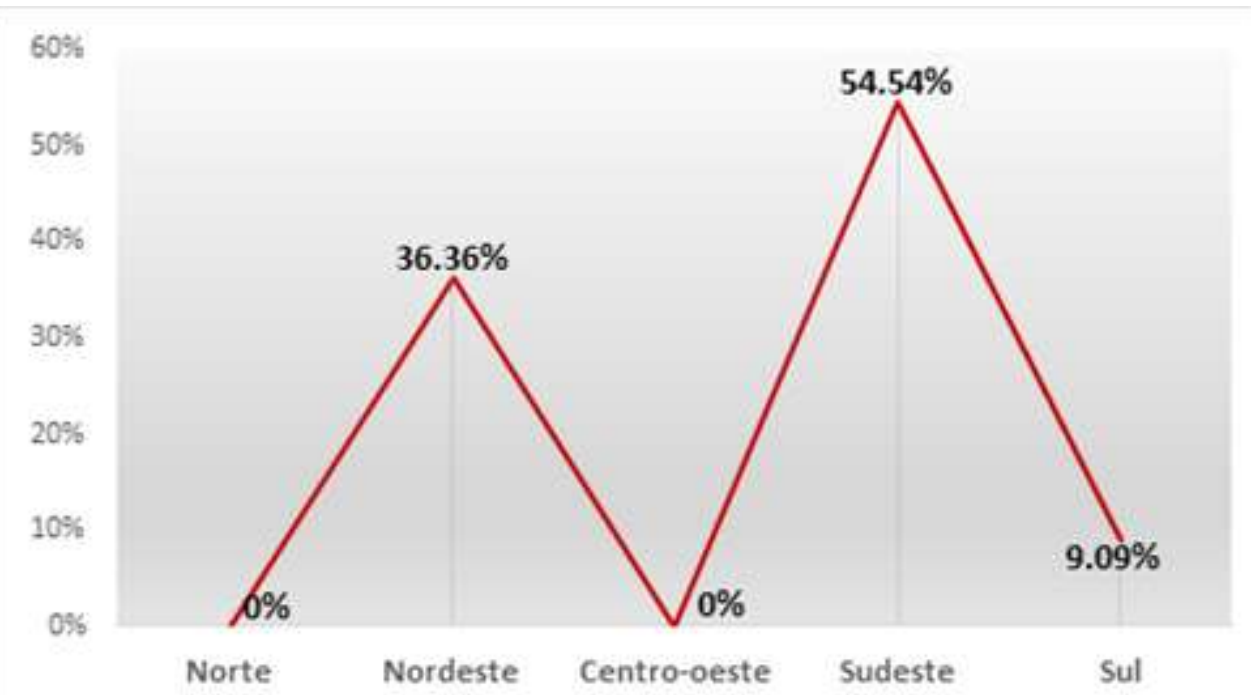

Gráfico 1: Distribuição dos estudos participantes da revisão, segundo as regiões em aue foram realizados.

Assim, diante da grande heterogenia de estudos e de concentração de casos de AIDS no país, novos estudos devem ser realizados em diferentes regiões, para que os gestores públicos tenham a atenção voltada para as regiões que mais necessitam de acesso ao tratamento e incentivo à adesão da TARV.

No que diz respeito à população, quase a totalidade dos artigos participantes desta revisão, investigaram adultos. No entanto, no Brasil, desde 2000 até junho de 2015, foram notificadas 92.210 de gestantes infectadas com o HIV, o que representa uma alta taxa de crescimento de novos casos nessa população nos últimos dez anos. Esse fato é importante devido a transmissão vertical do Vírus da Imunodeficiência Humana (HIV) da mãe para o bebê durante o período da gestação, nascimento ou aleitamento materno $^{12,13}$. Portanto, uma atenção especial deve ser dada as gestantes, as crianças e aos adolescentes.

A população idosa também merece um cuidado especial, tendo em vista que o uso dos antirretrovirais possibilitou o aumento da expectativa de vida para os portadores do HIV, portanto os indivíduos infectados estão envelhecendo, tornando-se idosos com AIDS ${ }^{14}$. Avanços tecnológicos na área médica, como medicamentos que melhoram o desempenho sexual e a reposição hormonal, possibilitaram o aumento da sexualidade entre adultos maiores de 50 anos. O não uso da camisinha entre esses indivíduos, também contribuiu para o aumento dos casos de $\operatorname{AIDS}^{15,16}$. Em 1998, foram notificados 626 novos casos de aids em pessoas acima dos 60 anos de idade no Brasil, já em 2012 esse número aumentou para $1.812^{14}$.

Diferentes métodos de verificação da adesão à TARV foram utilizados entre os artigos que compõe a presente revisão. Dentre eles, o registro da retirada dos medicamentos na farmácia foi o mais utilizado ${ }^{17-20}$. Esse método é realizado em centros especializados no atendimento de pacientes HIV soropositivos, que oferecem atendimento médico e/ou multiprofissional, e possuem uma rede própria de distribuição dos fármacos antirretrovirais. A verificação da 
adesão é feita a partir da data de retirada desses medicamentos, baseando-se na teoria de que os pacientes que buscam os medicamentos no tempo certo, estabelecido pela indicação médica, tendem a tomá-los mais corretamente do que aqueles que atrasam até mesmo no momento da retirada $^{21}$. A vantagem dessa metodologia é o fácil acesso a informação, tendo em vista a informatização do sistema de prontuários desses centros, no entanto, não é possível afirmar que os pacientes que retiraram a medicação na data certa, fazem o uso correto desses medicamentos, essa seria uma desvantagem desse tipo de verificação. Os autores que utilizaram essa metodologia apresentaram diferenças entre o período de acompanhamento dos pacientes, o tempo de atraso considerado para a retirada do fármaco e a forma de classificação da taxa de não adesão. A forma mais utilizada de classificação foi: retiradas regulares, irregulares e o abandono de tratamento.

Quadro 2: Distribuição dos estudos participantes da revisão, segundo os autores, a região onde foi realizado, a população participante, o método de verificação da adesão e a taxa de não adesão à TARV.

\begin{tabular}{|c|c|c|c|c|}
\hline Autores & $\begin{array}{l}\text { Região em } \\
\text { que foi } \\
\text { realizado } \\
\text { o estudo }\end{array}$ & $\begin{array}{l}\text { População } \\
\text { de estudo }\end{array}$ & $\begin{array}{l}\text { Método de verificação de adesão a } \\
\text { TARV }\end{array}$ & $\begin{array}{l}\text { Taxa de não adesão a } \\
\text { TARV }\end{array}$ \\
\hline $\begin{array}{l}\text { Gomes RR de FM; } \\
\text { Machado CJ;Acurcio F de A; } \\
\text { Guimarães MDC. }\end{array}$ & Sudeste & $\begin{array}{l}(\mathrm{n}=323) \\
\text { Adultos }\end{array}$ & Registro de dispensão dos ARV. & $\begin{array}{l}\text { Retirada irregular }(57,9 \%) \text { e } \\
\text { abandono do tratamento } \\
(30,3 \%) \text {, }\end{array}$ \\
\hline Trombini ES, Schermann LB. & Sul & $\begin{array}{l}(n=44) \\
\text { Crianças }(18 \\
\text { meses a } 13 \\
\text { anos }\end{array}$ & $\begin{array}{l}\text { Inquérito com o cuidador sobre os } \\
\text { medicamentos administrados nos últimos } 3 \\
\text { dias. Análise do prontuário médico. }\end{array}$ & $\begin{array}{l}18 \% \text { informaram que } 0 \\
\text { tratamento foi } \\
\text { interrompido algumas vezes. }\end{array}$ \\
\hline $\begin{array}{l}\text { *Rocha GM; Machado } \\
\text { CJ;AcurcioFdeA; } \\
\text { Guimarães MDC. }\end{array}$ & Sudeste & $\begin{array}{l}(\mathrm{n}=288) \\
\text { Adultos }\end{array}$ & \multicolumn{2}{|c|}{$\begin{array}{l}\text { Registros em prontuários médicos --Taxa de não adesão (22,9\%) } \\
\begin{array}{l}\text { Autorrelato -- Taxa de não adesão (31,9\%) } \\
\text { Registros de dispensação nas farmácias --Taxa de não adesão }(74,3 \%)\end{array}\end{array}$} \\
\hline $\begin{array}{l}\text { Leite LHM; Papa A; Valentini } \\
\text { RC. }\end{array}$ & Sudeste & $\begin{array}{l}(\mathrm{n}=80) \\
\text { Adultos }\end{array}$ & $\begin{array}{l}\text { Modelo MOTHIV questionário de } \\
\text { autopreenchimento contendo } 54 \text { itens }\end{array}$ & $\begin{array}{l}61,2 \% \text { apresentavam } \\
\text { problemas relacionados a } \\
\text { adesão ao tratamento. }\end{array}$ \\
\hline Felix G;Ceolim MF. & Sudeste & $\begin{array}{l}(\mathrm{n}=60) \\
\text { Mulheres } \\
\text { Adultas }\end{array}$ & $\begin{array}{l}\text { Aplicação do Questionário: Ambulatório de } \\
\text { Adesão ao Tratamento/AIDS (construído e } \\
\text { validado no Brasil). } \\
\text { Ingestão de } 95 \% \text { da terapêutica antirretroviral } \\
\text { prescrita, nos três dias anteriores à entrevista. }\end{array}$ & $\begin{array}{l}\text { Taxa de não adesão de } \\
35 \% \text {. }\end{array}$ \\
\hline $\begin{array}{l}\text { Camargo LA; Capitão CG; } \\
\text { Filipe EMV. }\end{array}$ & Sudeste & $\begin{array}{l}(\mathrm{n}=73) \\
\text { Adultos }\end{array}$ & $\begin{array}{l}\text { Registros de retirada de medicação da } \\
\text { farmácia. }\end{array}$ & $\begin{array}{l}\text { 50,6\% da população } \\
\text { estudada tiveram uma taxa } \\
\text { de não adesão à TARV } \\
\text { (menos de } 75 \% \text { de retirada } \\
\text { nas farmácias) }\end{array}$ \\
\hline $\begin{array}{l}\text { Jacques IdeJAA; Santana } \\
\text { JMde; Moraes DCdeA; Souza } \\
\text { AdeFM; } \\
\text { Abrão FMdaS; Oliveira RCde. }\end{array}$ & Nordeste & $\begin{array}{l}(\mathrm{n}=152) \\
\text { Adultos }\end{array}$ & $\begin{array}{l}\text { CEAT-VIH (Cuestionario para La Evaluación } \\
\text { De La Adhesión al Tratamiento Antirretroviral } \\
\text { en Personas } \\
\text { Com Infección por VIH y Sida) }\end{array}$ & $\begin{array}{l}24,34 \% \text { dos participantes } \\
\text { apresentavam média ou } \\
\text { baixa adesão. }\end{array}$ \\
\hline $\begin{array}{l}\text { Silva ACO; } \\
\text { Reis RK; NogueiraJÁ;Gir E. }\end{array}$ & Nordeste & $\begin{array}{l}(\mathrm{n}=314) \\
\text { Adultos }\end{array}$ & $\begin{array}{l}\text { CEAT-VIH (Cuestionario para La Evaluación } \\
\text { De la Adhesión al Tratamiento Antirretroviral } \\
\text { en Personas } \\
\text { Com Infección por VIH y Sida) }\end{array}$ & $\begin{array}{l}\text { 73,8\% apresentaram } \\
\text { adesão insuficiente. }\end{array}$ \\
\hline $\begin{array}{l}\text { Moraes DCdeA; } \text { Oliveira } \\
\text { RCde; } \\
\text { Costa SFG. }\end{array}$ & Nordeste & $\begin{array}{l}(\mathrm{n}=136) \\
\text { Homens } \\
\text { adultos }\end{array}$ & $\begin{array}{l}\text { CEAT-VIH (Cuestionario para La Evaluación } \\
\text { De La Adhesión al Tratamiento Antirretroviral } \\
\text { en Personas } \\
\text { Com Infección por VIH y Sida) }\end{array}$ & $\begin{array}{l}\text { 63,3\% apresentaram } \\
\text { adesão } \\
\text { "insuficiente/regular". }\end{array}$ \\
\hline $\begin{array}{l}\text { Tufano CS; Amaral RAdo; } \\
\text { Cardoso LRD;Malbergier A. }\end{array}$ & Sudeste & $\begin{array}{l}(\mathrm{n}=438) \\
\text { Adultos }\end{array}$ & $\begin{array}{l}\text { The Simplified Medication } \\
\text { Questionnaire (SMAQ) }\end{array}$ & $\begin{array}{l}\text { Taxa de não adesão } 46,3 \% \\
\text { (nos } 3 \text { meses antecedentes } \\
\text { a pesquisa) e } 27,2 \% \text { (na } \\
\text { última semana antecedente } \\
\text { a pesquisa). }\end{array}$ \\
\hline $\begin{array}{l}\text { Silva JAG; Dourado I; Brito } \\
\text { AMde; Silva CALda. }\end{array}$ & Nordeste & $\begin{array}{l}(\mathrm{n}=216) \\
\text { Pacientes } \\
\text { acima de } 13 \\
\text { anos. }\end{array}$ & $\begin{array}{l}\text { Registros de retirada de medicamentos na } \\
\text { farmácia. }\end{array}$ & $25 \%$ de taxa de não adesão. \\
\hline
\end{tabular}

Os autores Rocha et al. ${ }^{18}$ realizaram um estudo que investigou três diferentes metodologias para verificar a taxa de não adesão: o registro da retirada dos medicamentos na farmácia, já explicado anteriormente, a análise do prontuário médico e o autorrelato. A análise do prontuário médico foi feita por meio da avaliação das informações presentes nesses documentos, que serviram de base para a verificação da adesão, qualquer situação que indicasse não adesão ao tratamento (como por exemplo, deixar de tomar uma dose, pular algum dia, histórico de abandono com motivo registrado ou não) foram anotadas. Indivíduos cujos registros médicos não continham qualquer anotação de não-adesão durante o período de estudo foram considerados aderentes ao tratamento $^{18}$. No autorrelato sobre a administração dos antirretrovirais, os autores consideraram como boa adesão aqueles que tomaram $95 \%$ da medicação nos 3 dias que antecederam a pesquisa ${ }^{18}$. Esse método tem sido muito utilizado em pesquisas e também na prática clínica para verificar a adesão aos medicamentos. Trata-se de um método de baixo custo, flexível e rápido. Essa metodologia permite também aprofundar uma discussão com o usuário acerca dos motivos e dificuldades em relação ao tratamento $^{21}$

No caso de pacientes dependentes como as crianças, que ainda não podem se responsabilizar pela administração dos antirretrovirais, a técnica utilizada por Trombini, Schermann ${ }^{22}$, foi a entrevista com os responsáveis. As desvantagens em relação ao autorrelato e a entrevista se dão pelo fato do paciente, muitas vezes, superestimar a adesão com receio de decepcionar ou desagradar os profissionais de saúde ${ }^{21}$, além disso, as diferenças entre as metodologias empregadas em pesquisas que utilizam esse método podem, dificultar a comparação dos resultados encontrados ${ }^{23}$.

A literatura apresenta alguns questionários validados que foram desenvolvidos com a finalidade de verificar a taxa de adesão à TARV. Entre eles destaca-se o "Cuestionario 
para la Evaluación de La Adhesión al Tratamiento Antiretroviral (CEAT-VIH)", validado para o português por Remor et.al. ${ }^{24}$, que foi utilizado por três estudos que compõe a presente revisão ${ }^{25-27}$. Trata-se de um instrumento composto por 20 itens que abordam os principais fatores que podem interferir na adesão à TARV em pacientes adultos: a história de não adesão do paciente; a relação médico-paciente; as crenças do paciente a respeito da TARV; as expectativas sobre a eficácia terapêutica; o esforço do paciente para seguir o tratamento; a avaliação da gravidade dos efeitos colaterais da TARV para o paciente; o grau de satisfação com a medicação antirretroviral e o uso de estratégias para lembrar-se de tomar a medicação. Após a análise dos resultados a adesão pode ser classificada em boa, regular/insuficiente ou baixa ${ }^{24-27}$.

O questionário "Simplified Medication Adherence Questionnaire (SMAQ)" também foi utilizado em um dos artigos que compõe a presente revisão ${ }^{28}$. Este instrumento contém seis questões, sendo quatro com respostas dicotômicas de "sim" ou "não" e duas qualitativas nas quais se investiga o não uso da medicação na última semana e nos últimos três meses. No entanto, este questionário não foi validado no Brasil ${ }^{28}$. A vantagem em relação a utilização de questionários validados se dá pela possibilidade da comparação dos resultados.

Dentre os estudos avaliados, Leite et al. ${ }^{29}$ verificaram a adesão à TARV, por meio do modelo MOTHIV, que propõe um diagnóstico das motivações para o tratamento antirretroviral, centrado no paciente e nas suas condições de vida. Trata-se de um questionário que contem 54 itens divididos em três partes: a primeira, com 14 itens relacionados com as causas mais comuns para o não uso dos antirretrovirais; a segunda, composta por 22 itens, voltada para a avaliação dos sintomas e efeitos colaterais ligados ao HIV e seu tratamento; e a terceira, contendo uma lista com 18 fatores de stress relacionados às condições de vida, suporte social, mudanças na rotina e acontecimentos da vida que dificultaram a adesão nos últimos três meses. Para cada opção de resposta, atribui-se a pontuação zero para ausência do problema, e 1 para a presença do problema. A adesão pode ser classificada em boa ou irregular dependendo da pontuação das respostas ${ }^{29}$.

A partir das diferentes metodologias utilizadas pelos estudos selecionados para participar desta revisão, observase uma alta taxa de não adesão a TARV na população brasileira, os valores variaram de $18 \%$ a $74,3 \%$. Em 2007 , Bonolo et al. ${ }^{30}$, realizaram uma revisão de literatura sobre 0 tema da adesão à TARV, composta por artigos nacionais e internacionais, e encontraram uma variação de 5 à $67 \%$ de taxa de não adesão ao tratamento antirretroviral. Essa comparação demonstra que os valores encontrados na presente pesquisa, são piores e preocupantes, merecendo atenção especial dos gestores de saúde do país.

A efetividade da TARV depende diretamente da adesão do paciente, devendo este consumir pelo menos $95 \%$ dos medicamentos prescritos, para que a carga viral seja mantida indetectáve ${ }^{25}$ o que pode diminuir razoavelmente, a possibilidade de transmissão do vírus ${ }^{31}$. Segundo o Ministério da Saúde uma boa adesão ao tratamento significa tomar corretamente os medicamentos, seguir as doses corretas pelo tempo preestabelecido, além de fazer o acompanhamento médico no serviço de saúde especializado $^{19,32}$.

Muitos fatores podem interferir negativamente na adesão ao tratamento, entre eles destacam-se: perfil socioeconômico, sexo, idade, renda, escolaridade, uso de drogas, aceitação e nível de conhecimento da doença, sintomas psicológicos e/ou transtornos psiquiátricos, além da percepção de suporte social ${ }^{19}$.

A adesão pode diminuir à medida que a complexidade e a duração do tratamento aumentam. Mudanças nas atividades diárias, nos hábitos alimentares, no estilo de vida, bem como os efeitos colaterais dos medicamentos podem contribuir para a não adesão ${ }^{31}$.

Portanto, a adesão à terapia antirretroviral é um desafio para o setor público de saúde, é imprescindível monitorar as taxas de não adesão, bem como avaliar as dificuldades que levam os pacientes a desistência do tratamento, e dessa forma subsidiar ações e políticas públicas voltadas para o enfrentamento desse grave problema de saúde pública.

\section{CONCLUSÃO}

Houve um alto índice de não adesão à terapia antirretroviral nos estudos que participaram dessa revisão. Os estudos tem uma tendência a polarização em algumas regiões do país. A população participante dos estudos, em sua maioria era adulta.

O método para verificação da adesão mais utilizado foi a retirada do medicamento nas farmácias seguido pela aplicação do questionário Cuestionario para La Evaluación de La Adhesión al Tratamiento Antirretroviral en Personas com Infección por VIH y Sida.

Faz-se necessário, portanto, novos estudos acerca da temática da adesão à terapia antirretroviral, em diferentes regiões e populações, para que se possa conhecer melhor a realidade nacional a fim de investir esforços direcionados às regiões mais vulneráveis.

\section{REFERÊNCIAS}

1. Brasil. Ministério da Saúde. Secretaria de Vigilância em Saúde. Departamento de DST, Aids e Hepatites Virais. Boletim Epidemiológico - Aids e DST. Brasília 2015.

2. Brasil. LEI No 7.670, DE 8 DE SETEMBRO DE 1988. Brasília 1988. (Disponível em: https://www.planalto.gov.br/ccivil_03/leis/17670.htm)

3. Brasil. LEI No 12.984, DE 2 DE JUNHO DE 2014. Brasília 2014. (Disponível em: http://www.planalto.gov.br/ccivil_03/_Ato20112014/2014/Lei/L12984.htm)

4. Nunn AS, Fonseca EM, Bastos FI, Gruskin S, Salomon JA. Evolution of Antiretroviral Drug Costs in Brazil in the Context of Free and Universal Access to AIDS Treatment. PLoS Med. 2007; 4(11): e305.

5. Schaurich D, Coelho DF, Motta MGC. A cronicidade no processo saúde-doença: repensando a epidemia da AIDS após os anti-retrovirais. Rev Enferm UERJ. 2006;14(3):455-62.

6. Drachler MdL, Drachler CW, Teixeira LB, de Carvalho Leite JC. The Scale of Self-Efficacy Expectations of Adherence to Antiretroviral Treatment: A Tool for Identifying Risk for Non-Adherence to Treatment for HIV. PLoS ONE. 2016; 11(2):e-0147443.

7. Baer M, Roberts J. Complex HIV treatment regimens and patient quality of life. Can Psychol. 2002; 43(2):115-21.

8. Seidl EMF, Melchíades A, Farias V, Brito A. Pessoas vivendo com HIV/AIDS: variáveis associadas à adesão ao tratamento anti-retroviral. Cad Saúde Pública. 2007; 23(10):2305-16.

9. Souza MT, Silva MD, Carvalho R. Integrative review: 
what is it? How to do it? Einstein. 2010; 8(1):102-6.

10. Sousa AIA de, Pinto Júnior VL. Análise espacial e temporal dos casos de aids no Brasil em 1996-2011: áreas de risco aumentado ao longo do tempo. Epidemiol Serv Saúde. 2016; 25(3):467-76.

11. Teixeira TRA, Gracie R, Malta MS, Bastos FI. Social geography of AIDS in Brazil: identifying patterns of regional inequalities. Cad Saúde Pública. 2014; 30(2):259-71.

12. Guanabara MAO, Araujo MAL, Barros VL, Gondima OS, Pinheiro PMR, Oliveira FA. Gestantes com HIV/Aids acompanhadas em serviços públicos. Rev Enferm UFPI. 2014; 3(2):25-32.

13. Felix G, Ceolim MF. O perfil da mulher portadora de HIV/AIDS e sua adesão à terapêutica antirretroviral. Rev esc enferm USP. 2012; 46(4): 884-91.

14. Affeldt AB, Silveira MF, Barcelos RS. Perfil de pessoas idosas vivendo com HIV/AIDS em Pelotas, sul do Brasil, 1998 a 2013. Epidemiol Serv Saúde. 2015; 24(1):79-86.

15. Bertoncini BZ, Moraes KS, Kulkamp IC. Comportamento sexual em adultos maiores de 50 anos infectados pelo HIV. J Bras Doenças Sex Trans. 2007; 19(2):75-9.

16. Alencar RA, Ciosak SI. O diagnóstico tardio e as vulnerabilidadesdos idosos vivendo com HIV/aids. Rev esc enferm USP. 2014; 49(2):229-35.

17. Gomes RRFM, Machado CJ, Acurcio FA, Guimarães MDC. Utilização dos registros de dispensação da farmácia como indicador da não-adesão à terapia antiretroviral em indivíduos infectados pelo HIV. Cad Saúde Pública. 2009; 25(3):495-506.

18. Rocha GM, Machado CJ, Acurcio FA, Guimarães MDC. Monitoring Adherence to antiretroviral treatment in Brazil: an urgent challenge. Cad Saúde Pública. 2011; 27(1):67-78.

19. Camargo LA, Capitão CG, Filipe EMV. Mental health, family support and treatment adherence: associations in the context of HIV/aids. Psico-USF. 2014;19(2):221-32.

20. Silva JAG, Dourado I, Brito AM, Silva CAL. Fatores associados à não adesão aos antirretrovirais em adultos com AIDS nos seis primeiros meses da terapia em Salvador, Bahia, Brasil. Cad Saúde Pública. 2015; 31(6):1188-98.

21. Polejack L, Seidl EMF. Monitoramento e avaliação da adesão ao tratamento antirretroviral para HIV/aids: desafios e possibilidades. Ciênc saúde coletiva. 2010; 15(supl 1):1201-08.

22. Trombini ES, Schermann LB. Prevalência e fatores associados à adesão de crianças na terapia antirretroviral em três centros urbanos do sul do Brasil. Ciênc saúde coletiva. 2010; 15(2):419-25.

23. Sankar A, Golin C, Simoni J, Luborsky M, Pearson C. How Qualitative Methods Contribute to Understanding Combination Antiretroviral Therapy Adherence. J Acquir Immune Defic Syndr. 2006; 43(Suppl 1):S54-68.

24. Remor E, Milner-Moskovics J, Preussler G. Adaptação brasileira do "Cuestionario para la Evaluación de la Adhesión al Tratamiento Antiretroviral" Rev Saúde Pública. 2007;41(5):685-94.

25. Jacques IJAA, Santana JM, Moraes DCA, Souza AFM, Abrão FMS, Oliveira RC. Avaliação da Adesão à Terapia Antirretroviral entre Pacientes em Atendimento Ambulatorial. Rev bras cienc saúde. 2014; 18(4):303-8.

26. Silva ACO, Reis RK, Nogueira JA, Gir E. Quality of life, clinical characteristics and treatment adherence of people living with HIV/AIDS. Rev Latino-Am Enfermagem. 2014; 22(6):994-1000.

27. Moraes DCA, Oliveira RC, Costa SFG. Adesão de homens vivendo com HIV/AIDS ao tratamento antirretroviral. Esc Anna Nery. 2014; 18(4):676-81.

28. Tufano CS, Amaral RA, Cardoso LRD, Malbergier A. The influence of depressive symptoms and substance use on adherence to antiretroviral therapy. A crosssectional prevalence study. Sao Paulo Med J. 2015; 133(3):179-86

29. Leite LHM, Papa A, Valentini RC. Insatisfação com imagem corporal e adesão à terapiaantirretroviral entre indivíduos com HIV/AIDS. Rev Nutr. 2011; 24(6):87382.

30. Bonolo PF, Gomes RRFM, Guimarães MDC. Adesão à terapêutica anti-retroviral (HIV/AIDS): fatores associados e medidas de adesão. Epidemiol Serv Saúde. 2007; 16(4):267-78.

31. Rossi OS, Pereira PPG. O remédio é o menor dos problemas: seguindo redes na adesão ao tratamento de AIDS. Saúde Soc São Paulo. 2014; 23(2): 484-95.

32. Brasil. Ministério da Saúde. Secretaria de Vigilância em Saúde Programa Nacional de DST e AIDS. Manual de adesão ao tratamento para pessoas vivendo com HIV e AIDS. 2007.

\section{CONFLITO DE INTERESSES}

Os autores declaram não haver conflitos de interesse.

\section{AUTOR PARA CORRESPONDÊNCIA}

\section{Cléa Adas Saliba Garbin}

cgarbin@foa.unesp.br

Submetido em 04/11/2016 Aceito em 05/12/2016 